\title{
Herramienta de detección de apnea del sueño obstructiva
}

\author{
A tool to screen patients for obstructive sleep apnea
}

\section{Objetivo}

Desarrollar y validar un cuestionario para identificar pacientes con apnea del sueño obstructiva (ASO).

Diseño, lugar, pacientes y descripción de las pruebas

Corte transversal llevado a cabo en dos hospitales canadienses.

\author{
Chung F y col. Anesthesiology. 2008; 108(5):812-21.
}

El cuestionario (ver tabla 1) fue desarrollado* sobre 2467 pacientes prequirúrgicos $(27,5 \%$ de alto riesgo de ASO) y validado* en otros 177, comparándolo con el índice de apneahipopnea (IAH) y con una polisomnografía monitorizada (prueba diagnóstica de referencia costosa y que requiere de una noche en el laboratorio).

Tabla 1: Descripción de los cuestionarios STOP y STOP-BANG.

\begin{tabular}{|c|c|}
\hline $\begin{array}{c}\text { STOP } \\
\text { Snoring }(\mathrm{S}) \text {, tired }(\mathrm{T}) \text {, observed }(\mathrm{O}) \text {, pressure }(\mathrm{P})\end{array}$ & $\begin{array}{c}\text { STOP-BANG } \\
\text { STOP + BMI (B) age (A) neck circumference (N) gender (B) }\end{array}$ \\
\hline $\begin{array}{l}\text { 1. ¿Ronca usted fuerte (más fuerte que hablando o lo suficiente como para que se le oiga } \\
\text { a través de una puerta cerrada)? } \\
\text { 2. ¿Se siente cansado, fatigado o somnoliento durante el día? } \\
\text { 3. ¿Le ha observado alguien dejar de respirar durante el sueño? } \\
\text { 4. ¿Tiene usted o está siendo tratado por hipertensión arterial? } \\
\text { Dos o más respuestas afirmativas implican alto riesgo }\end{array}$ & $\begin{array}{l}\text { Las cuatro anteriores más: } \\
\text { 5. ¿Indice de masa corporal (IMC) mayor de } 35 \mathrm{~kg} / \mathrm{m}^{2} \text { ? } \\
\text { 6. ¿Edad mayor de } 50 \text { años? } \\
\text { 7. ¿Circunferencia del cuello mayor de } 40 \mathrm{~cm} \text { ? } \\
\text { 8. ¿Sexo masculino? } \\
\text { Tres o más respuestas afirmativas implican alto riesgo }\end{array}$ \\
\hline
\end{tabular}

\section{Resultados principales}

En el grupo de validación* el índice apnea-hipopnea fue $20 \pm 6$, resumiéndose las principales características operativas de los cuestionarios en la tabla 2.

Tabla 2: características operativas de los cuestionarios para cada nivel de índice apnea hipopnea (IAH).

\begin{tabular}{|c|c|c|c|c|c|c|}
\hline \multirow{2}{*}{ Resultados } & \multicolumn{3}{|c|}{ Guestionario STOP } & \multicolumn{3}{|c|}{ Cuestionario STOP-BANG } \\
\hline & IAH $>5$ & IAH $>15$ & IAH $>30$ & IAH $>\mathbf{5}$ & IAH $>15$ & IAH $>\mathbf{3 0}$ \\
\hline Sensibilidad (\%) & 66 (56 a 74$)$ & $74(62$ a 84$)$ & 80 (64 а 91) & 84 (76 a 90$)$ & 93 (84 a 98$)$ & 100 (91 a 100) \\
\hline Especificidad (\%) & 60 (46 a 73$)$ & 53 (43 a 63) & 49 (40 a 63$)$ & 56 (42 a 70$)$ & 43 (34 a 53$)$ & $37,0$ (29 а 46$)$ \\
\hline VPP (\%) & 78 (69 a 86$)$ & 51 (41a 61) & 30 (22 a 40) & 81 (73 a 87) & 52 (42 a 61$)$ & 31 (23 a 40$)$ \\
\hline VPN (\%) & 44 (33 a 56$)$ & 76 (65 a 85$)$ & $89(80$ a 95$)$ & 61 (46 a 74$)$ & $90,2$ (79 а 97$)$ & 100 (93 a 100) \\
\hline CPP & $1,6(1,2$ a 2,4$)$ & $1,6(1,3$ a 2$)$ & $1,6(1,3$ a 2$)$ & $1,9(1,4$ a 2,7$)$ & $1,6(1,4$ a 2$)$ & $1,6(1,43$ a 1,8$)$ \\
\hline
\end{tabular}

VPP: Valor Predictivo Positivo. VPN: Valor Predictivo Negativo. CPP: Coeficiente de probabilidad positivo*.

\section{Conclusiones}

La sensibilidad de los cuestionarios es alta, especialmente en pacientes con ASO moderada a severa.

Palabras clave: apnea del sueño obstructiva, cuestionario, regla de predicción clínica. Key words: obstructive sleep apnea, survey, clinical prediction rule. Fuente de financiamiento: no referida.

Ver glosario*

\section{Comentario}

La ASO es el trastorno del sueño más frecuente ${ }^{1}$ y afecta del 2 al $26 \%$ de la población general' 2 .

Se asocia a somnolencia diurna, lesiones no intencionales, ronquidos, hipertensión arterial, enfermedad cardiovascular, peor calidad de vida $^{3-4}$ y mayor riesgo de eventos adversos perioperatorios $^{6-7}$, siendo subdiagnosticados ${ }^{5}$ más del $80 \%$ de los casos moderados o severos.

\section{Conclusiones del comentador}

La prevalencia de de ASO en el subgrupo que aceptó hacerse la polisomnografía -posiblemente autoseleccionados por trastornos del sueño- fue alta, lo que sobrestimaría las características operativas del cuestionario. Sin embargo, en contextos como el evaluado -pacientes quirúrgicos no oncológicos-, su buen VPN y aceptable especificidad permitirían el uso de estas herramientas para rastreo, especialmente para descartar ASO.

Agustín Ciapponi [ Servicio de Medicina Familiar y Comunitaria. Hospital Italiano de Buenos Aires. agustín.ciapponi@hospitalitaliano.org.ar ]

\section{Referencia}

1. Kryger MH: Diagnosis and management of sleep apnea syndrome. Clin Cornerstone 2000; 2:39-47.

2. Young T y col. The gender bias in sleep apnea diagnosis: Are women missed because they have different symptoms? Arch Intern Med 1996; 156:2445-51

3. Turkington $\mathrm{P}$ y col. Relationship between obstructive sleep apnoea, driving simulator performance, and risk of road traffic accidents. Thorax 2001; 56:800-5

4. Shahar E y col. Sleep-disordered breathing and cardiovascular disease: Cross-sectional results of the sleep heart health study. Am J Respir Crit Care Med 2001; 163:19-25 5. Gupta R y col. Postoperative complications in patients with obstructive sleep apnea syndrome undergoing hip or knee replacement: A case-control study. Mayo Clin Proc 2001; 76:897-905

6. Liao P y col. Respiratory complications among obstructive sleep apnea (OSA) patients who underwent surgery (abstract). Sleep 2007; 30 (suppl):0582.

7. Young T y col. Estimation of the clinically diagnosed proportion of sleep apnea syndrome in middle-aged men and women. Sleep 1997; 20:705-6 